\title{
Radiological Findings in COVID-19 and Adaptive Approaches for Radiology Departments: Literature Review and Experience Sharing
}

\author{
LFH Ng ${ }^{1}$, HHC Tsang ${ }^{2}$, FHY Wong ${ }^{3}$, MWC Law ${ }^{4}$, WH Chong ${ }^{5}$, CHN Ho ${ }^{6}$, JKJ Fung ${ }^{7}$, \\ CCY Chan ${ }^{8}$, LSK Li ${ }^{1}$, KT Wong ${ }^{4}$, JCX Chan ${ }^{5}, \mathrm{SHY} \mathrm{Lam}^{3}$, KH Wong ${ }^{9}$, PL Kwok ${ }^{7}, \mathrm{~L} \mathrm{Xu}^{2}$, \\ TKK Lai ${ }^{6}$, KK Cheng ${ }^{8}$, TYW Hon ${ }^{10}$, JYH Hui' ${ }^{10}$, SKY Kwok ${ }^{5}$, JKF Ma ${ }^{1}$ \\ ${ }^{\prime}$ Department of Radiology, Princess Margaret Hospital, Laichikok, Hong Kong \\ ${ }^{2}$ Department of Radiology and Organ Imaging, Queen Elizabeth Hospital, Jordan, Hong Kong \\ ${ }^{3}$ Department of Radiology, Queen Mary Hospital, Pokfulam, Hong Kong \\ ${ }^{4}$ Department of Imaging and Interventional Radiology, Prince of Wales Hospital, Shatin, Hong Kong \\ ${ }^{5}$ Department of Radiology, Tuen Mun Hospital, Tuen Mun, Hong Kong \\ ${ }^{6}$ Department of Radiology, Tseung Kwan O Hospital, Tseung Kwan O, Hong Kong \\ ${ }^{7}$ Department of Radiology, Pamela Youde Nethersole Eastern Hospital, Chai Wan, Hong Kong \\ ${ }^{8}$ Department of Diagnostic and Interventional Radiology, Kwong Wah Hospital, Yaumatei, Hong Kong \\ ${ }^{9}$ Department of Radiology, North District Hospital, Sheung Shui, Hong Kong \\ ${ }^{10}$ Department of Radiology, United Christian Hospital, Kwun Tong, Hong Kong
}

\begin{abstract}
Radiological investigations play an important role in the treatment course of patients with coronavirus disease 2019 (COVID-19) and radiologists should be familiar with the imaging characteristics. Being an integral component of the healthcare system, radiology departments have made adaptations to enhance infection control and strengthen the service. In this article, we review the radiological features of COVID-19 on chest radiography and computed tomography, and share experiences on the adaptive approach of radiology departments amidst the COVID-19 pandemic.
\end{abstract}

Key Words: Coronavirus; COVID-19; Radiography; Severe acute respiratory syndrome coronavirus 2

\footnotetext{
Correspondence: Dr LFH Ng, Department of Radiology, Princess Margaret Hospital, Laichikok, Hong Kong

Email:ngphonehim@gmail.com
}

Submitted: 24 Apr 2020; Accepted: 13 May 2020.

Contributors: LFHN, JCXC, SKYK and JKFM designed the study. All authors contributed to the acquisition of data. LFHN and JCXC analysed the data and drafted the manuscript. All authors had critical revision of the manuscript for important intellectual content. All authors had full access to the data, contributed to the study, approved the final version for publication, and take responsibility for its accuracy and integrity.

Conflicts of Interest: All authors have disclosed no conflicts of interest.

Funding/Support: This research received no specific grant from any funding agency in the public, commercial, or not-for-profit sectors. 


\title{
中文摘要
}

\section{9冠狀病毒病的放射學發現和放射科的調整處理方法：文獻綜述和 經驗分享}

\author{
吳豐謙、曾凱晴、黃皓源、羅穎聰、莊永豪、何影相、馮喬政、陳卓忻、李思琪、 \\ 黃嘉德、陳積聖、林曉燕、黃健開、郭寶琳、徐璐、賴國強、鄭加勁、韓予偉、許懿馨、 \\ 郭啓欣、馬嘉輝 \\ 放射學檢查在2019冠狀病毒病（COVID-19）患者的治療過程中起著重要作用, 放射科醫生應熟悉其 \\ 成像特徵。作為醫療系統不可或缺的組成部分, 放射科已經做出調整以增強感染控制和提高服務質 \\ 量。本文回顧COVID-19在胸部X光和電腦斷層掃描上的放射學特徵, 並分享COVID-19大流行中放射 \\ 科調整處理方法的經驗。
}

\section{INTRODUCTION}

In late December 2019, the World Health Organization (WHO) was informed of a cluster of cases of pneumonia of unknown cause detected in Wuhan City, Hubei Province, China. Subsequent deep sequencing analysis from lower respiratory tract samples indicated a novel coronavirus as the causative organism. The International Committee on Taxonomy of Virus named the virus severe acute respiratory syndrome coronavirus 2 (SARS-CoV-2). ${ }^{1}$ In February 2020, the WHO officially named the disease coronavirus disease 2019 (COVID-19). The disease spread rapidly, resulting in an epidemic throughout China, followed by other countries around the world. On 11 March 2020, the WHO officially declared COVID-19 a pandemic. ${ }^{2}$ According to data published by the WHO on 19 April 2020 (10:00 CEST), more than two million cases of COVID-19 have been confirmed worldwide, including more than 150000 deaths from the disease. ${ }^{3}$

The diagnosis of SARS-CoV-2 infection largely depends on clinical and epidemiological history with subsequent laboratory confirmation by realtime reverse-transcription polymerase chain reaction (RT-PCR) tests. Radiological investigations also play an important role in the treatment course of patients with COVID-19. To provide radiologists with the latest information, herein we review the radiological investigations and imaging features of COVID-19 and share the experiences of radiology departments in Hong Kong.

\section{RADIOLOGICAL INVESTIGATIONS FOR COVID-19 Chest Radiography}

Plain chest radiography (CXR) is readily available and is commonly performed in patients presenting with respiratory symptoms of various causes. In patients with COVID-19, an initial CXR helps not only to detect features of pneumonia, but also to identify or rule out differential diagnoses, such as pneumothorax or heart failure. In countries facing resource constraints with limited availability and long turnaround time of RT-PCR tests, CXR is recommended for medical triage of patients who present with moderate to severe clinical features and a high pre-test probability of COVID- $19 .^{4}$

Depending on the clinical setting and time of presentation, CXR abnormalities are reported in $33.3 \%$ to $95 \%$ of patients with COVID-19. ${ }^{5-10}$ In a study on 64 patients with RT-PCR-confirmed SARS-CoV-2 infection from four hospitals in Hong Kong, $69 \%$ of patients demonstrated abnormalities on baseline CXR, with $80 \%$ of patients exhibiting CXR abnormalities at some point during their disease course. ${ }^{7}$ Because the sensitivity of CXR could be related to the time of imaging and severity of pulmonary involvement, a normal CXR cannot exclude the diagnosis of COVID-19 (Figure 1a).

For patients with confirmed SARS-CoV-2 infection, CXR has the advantage of being portable for imaging within the isolation rooms, thereby reducing the risk of disease transmission during patient transportation. 
Portable CXR is invaluable to assess disease progression and rule out complications. However, in patients with confirmed SARS-CoV-2 infection, CXR should only be performed when there is appropriate clinical need, such as when there is clinical deterioration, rather than as a daily routine. Avoidance of non-value-added imaging is particularly important in patients with COVID-19, to minimise radiation exposure, to reduce the risk of disease transmission to radiographers, and to conserve personal protective equipment.

\section{Imaging Features on Chest Radiography}

The most common findings on CXR are consolidation and ground-glass opacities (GGOs), found more often in a bilateral, peripheral, and lower zone distributions (Figure 1b) ${ }^{5,7}$ Pleural effusion is uncommon, found only in 3\% of patients. ${ }^{7}$ A study in Hong Kong found that CXR findings reach peak severity at 10 to 12 days from symptom onset (Figure 2). ${ }^{7}$ This is in concordance with the earlier peak severity at 6 to 11 days from symptom onset, ${ }^{11}$ reported for computed tomography (CT), which is more sensitive. This is also in concordance with the clinical course; sepsis and acute respiratory distress syndrome have been found to occur at 9 to 12 days from symptom onset. ${ }^{12}$ Nevertheless, CXR findings in COVID-19 are not organism-specific and can overlap with other viral infections including influenza, severe acute respiratory syndrome (SARS), and Middle East respiratory syndrome (MERS). Furthermore, co-infection with other respiratory pathogens can occur in patients with confirmed SARS-CoV-2 infection. ${ }^{13}$

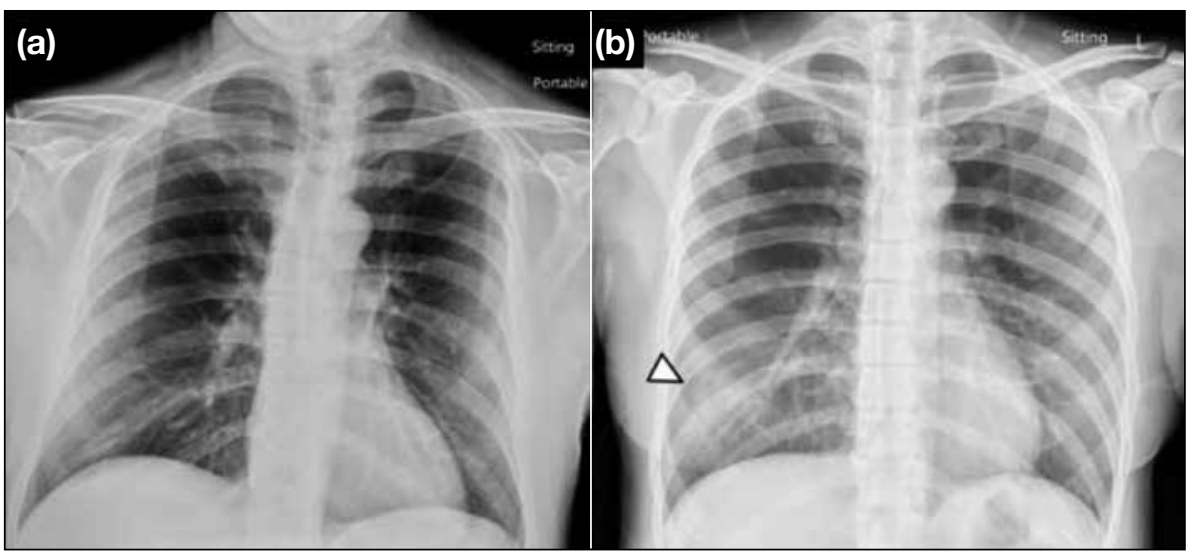

Figure 1. (a) Plain chest radiograph of a 39-year-old patient with coronavirus disease 2019, taken on day 5 after reverse-transcription polymerase chain reaction test confirming severe acute respiratory syndrome coronavirus 2 infection. (b) Right lower zone consolidation (arrowhead) on plain chest radiograph in a 52-year-old patient with coronavirus disease 2019, taken on day 6 after reverse-transcription polymerase chain reaction test confirming severe acute respiratory syndrome coronavirus 2 infection.

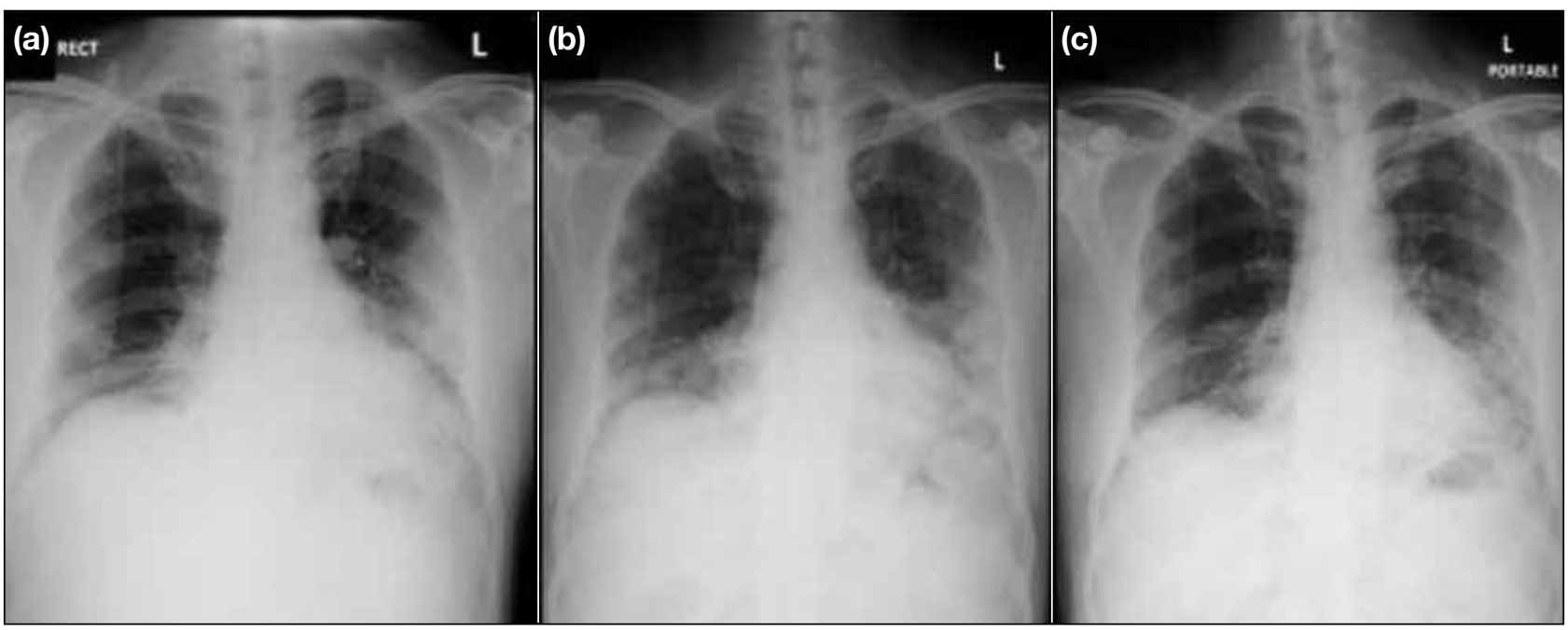

Figure 2. Serial plain chest radiographs of a 51-year-old patient with coronavirus disease 2019, taken (a) 1 day, (b) 12 days, and (c) 18 days after symptom onset. Extent of consolidation is largest on day 12 . 


\section{Computed Tomography}

Compared with CXR, chest CT is less readily available and results in greater radiation exposure. Nevertheless, it provides superior delineation of the pulmonary involvement caused by COVID-19. Chest CT is reported to have higher sensitivity for the diagnosis of SARS-CoV-2 infection when compared with initial RT-PCR test results. The sensitivity of chest CT has been reported in various studies as 98\%, 97\%, 93\%, and $61 \% \cdot{ }^{14-17}$ However, chest CT has limited specificity as low as $25 \%,{ }^{15}$ and should not be used as the sole method for the diagnosis of COVID-19. Discordance between results of RT-PCR and chest CT is commonly encountered and may lead to diagnostic confusion. ${ }^{18,19}$ A multidisciplinary approach, involving a combination of clinical history, clinical manifestations, imaging features, and laboratory results, is therefore desirable to achieve a timely and accurate diagnosis. According to a multinational consensus statement from the Fleischner Society, chest CT is not routinely indicated as a screening test for COVID-19 in asymptomatic individuals. Imaging is also not indicated for patients with mild features of COVID-19, unless they are at risk for disease progression. In contrast, chest CT is indicated for patients showing worsening respiratory status and/or moderate to severe features of COVID-19. ${ }^{4}$

CT scans can be used to evaluate complications related to COVID-19. Pleural effusion, multiple tiny pulmonary nodules, and mediastinal lymphadenopathy are atypical in COVID-19 pneumonia and their appearance should raise concern for bacterial superinfection or alternative diagnosis. ${ }^{20}$ In addition, CT can readily detect other complications especially in patients with COVID-19 who have been admitted under intensive care unit, to confirm or rule out pulmonary embolism during the acute setting.

\section{Imaging Features on Chest Computed Tomography}

The cardinal hallmark of COVID-19 pneumonia on chest CT is bilateral GGOs with or without consolidation in peripheral and posterior lungs (Figures 3 and 4). ${ }^{11,21}$ The GGOs often have rounded morphology (Figure 5) or are present with interlobular septal thickening and intralobular lines creating a "crazy-paving" pattern (Figure 6). ${ }^{20}$ GGO together with small areas of consolidation may suggest an organising pneumonia pattern of lung injury. ${ }^{21}$

Aligned by the Hong Kong College of Radiologists and with the cooperative effort of all radiology centres managed by the Hong Kong Hospital Authority, chest CT images of patients with confirmed SARS-CoV-2 infection scanned from 22 January 2020 to 16 April 2020 in six public hospitals in Hong Kong were reviewed

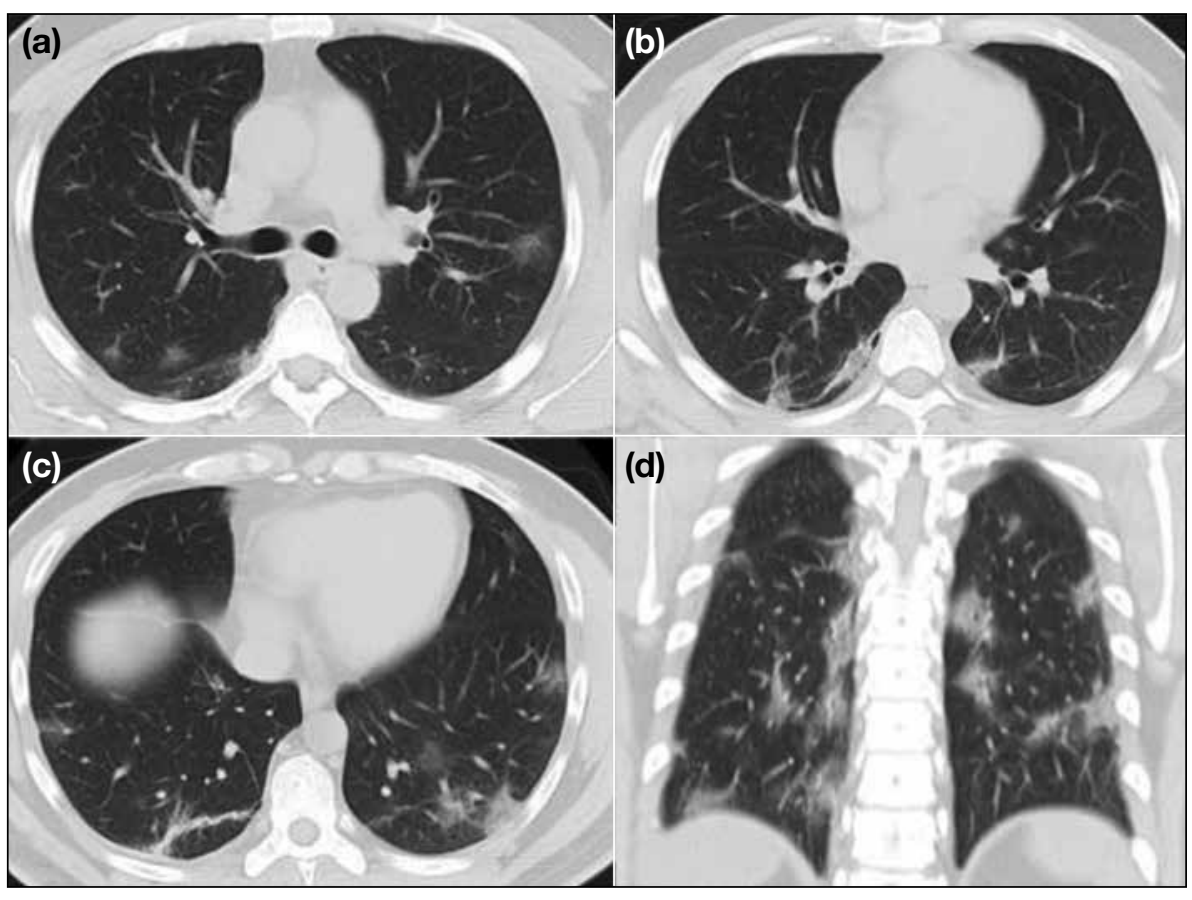

Figure 3. Typical computed tomography imaging features in a 39-year-old man with coronavirus disease 2019. (a-c) Unenhanced, thin-section axial images and (d) coronal reformatted image of the lungs 4 days after the onset of symptoms showing bilateral, multifocal peripheral ground-glass opacities with predominance in bilateral lower lobes. Linear opacities are also seen which are likely due to subsegmental atelectasis at this early stage of disease. 

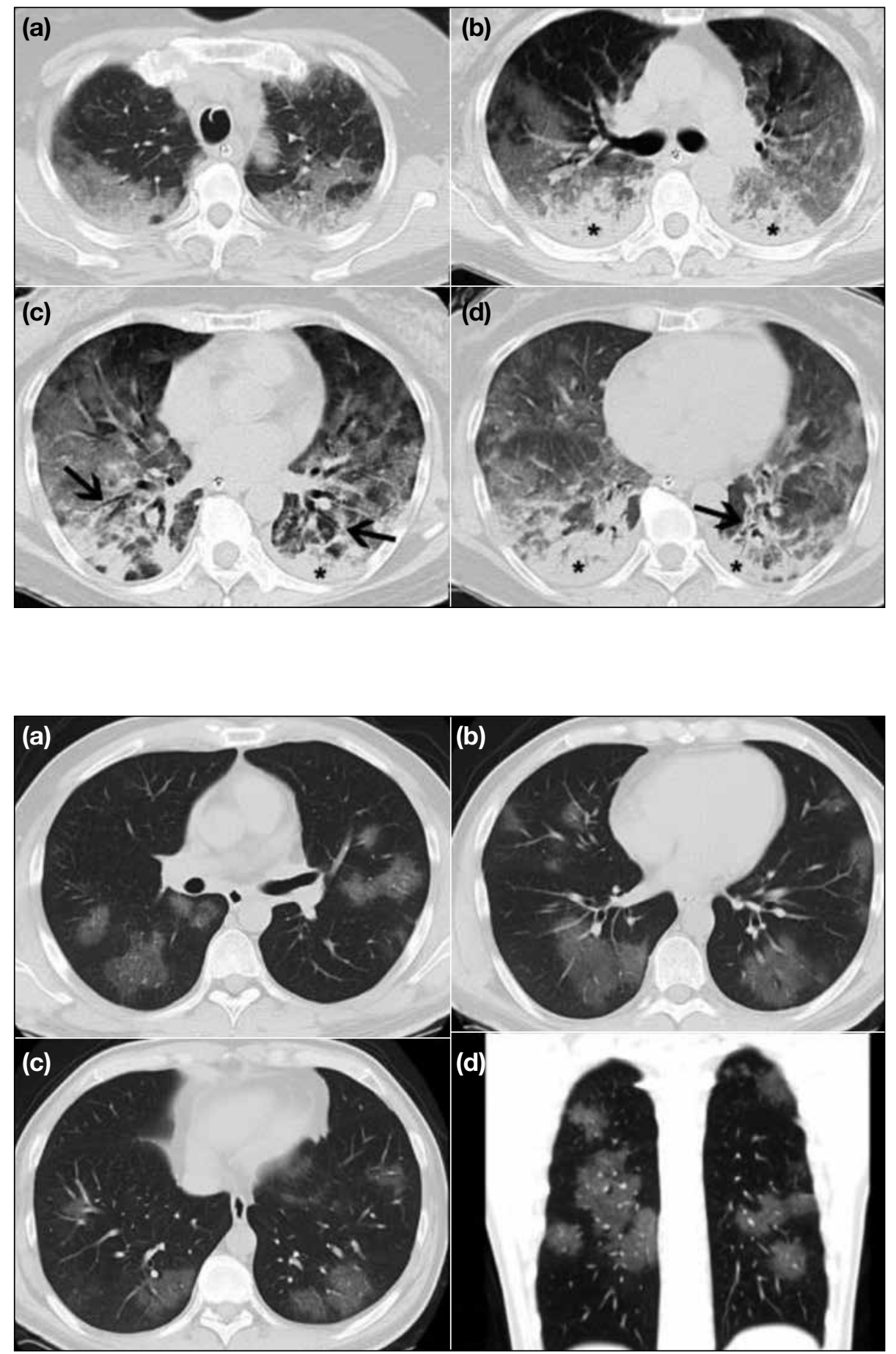

Figure 4. Typical computed tomography features in a 65-year-old woman with coronavirus disease 2019. (a-d) Unenhanced, thinsection axial images of the lungs 14 days after the onset of symptoms show bilateral, multifocal peripheral ground-glass opacities with consolidations (asterisks). Also note the presence of bronchial dilatation and wall thickening (arrows).
Figure 5. Typical computed tomography imaging features in a 39-year-old man with coronavirus disease 2019. (a-c) Unenhanced, thin-section axial images and (d) coronal reformatted image of the lungs 5 days after the onset of symptoms showing bilateral, multifocal rounded and peripheral ground-glass opacities. by qualified radiologists of the Hong Kong College of Radiologists (Table 1). Total 110 patients with RT-PCRconfirmed SARS-CoV-2 infection were included in this evaluation, $97(88.2 \%)$ of whom had abnormal findings on their first chest CT after admission. Among the radiological findings, GGO (Figures 3 to 7) was the most common, occurring in $95(97.9 \%)$ patients. Consolidation (Figure 4) was recorded in $57(58.8 \%)$ patients, septal thickening in 28 (28.9\%) patients, and reticular/ linear densities in 18 (18.6\%) patients. Bronchial wall thickening and dilatation (Figure 4) were less common, present in $13(13.4 \%)$ patients. Reticulonodular opacities and nodules were rare, and were found in the same one $(1.0 \%)$ patient. None of the patients had nodules with cavitation. In terms of lesion distribution, 94 (96.9\%) patients had predominantly peripheral distribution, 


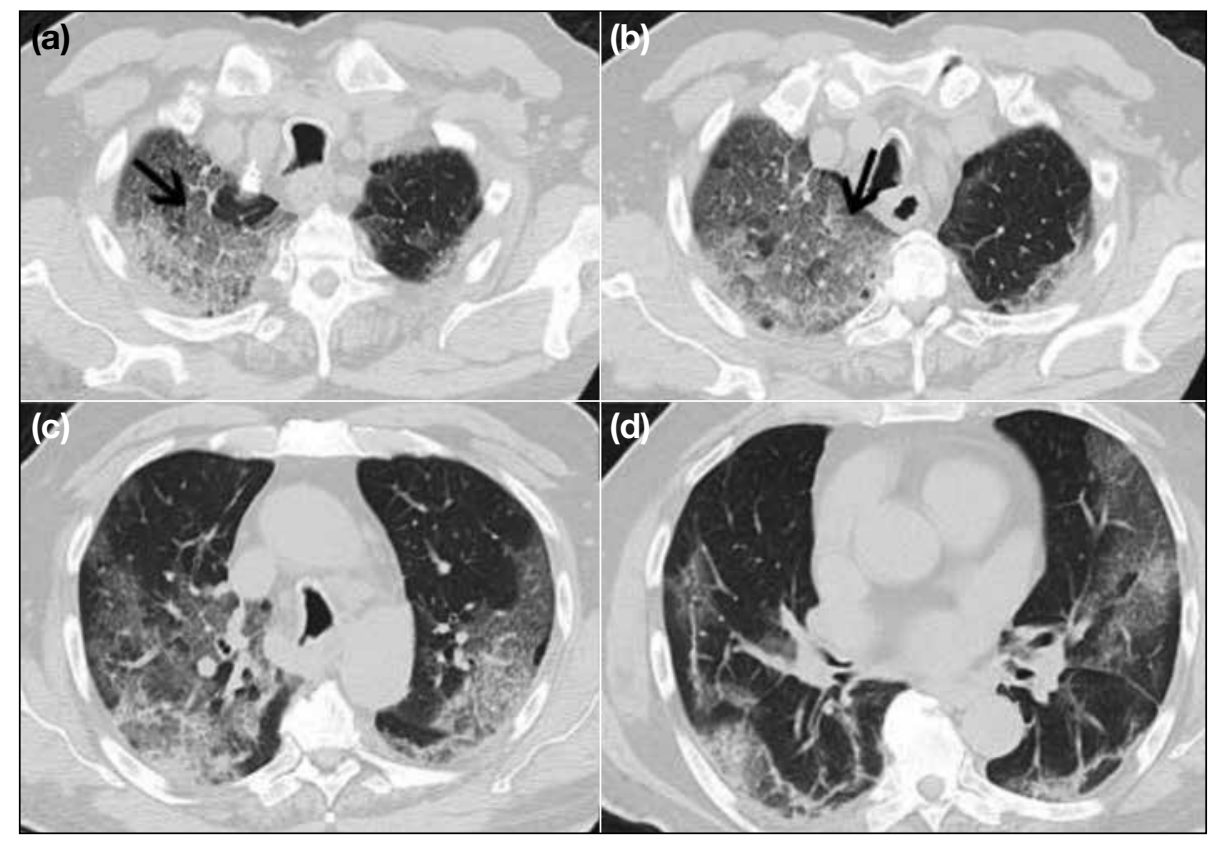

Figure 6. Typical computed tomography imaging features in a 75 -year-old man with coronavirus disease 2019. (a-d) Unenhanced, thin-section axial images of the lungs 9 days after the onset of symptoms show bilateral, multifocal peripheral ground-glass opacities with superimposed interlobular septal thickening and intralobular lines giving rise to "crazy-paving" appearance (arrows).
Table 1. Summary of 110 patients with chest computed tomography scanned from 22 January 2020 to 16 April 2020 in six public hospitals. ${ }^{\star}$

\begin{tabular}{lc}
\hline & Value \\
\hline Age, mean (range) & $47(12-96)$ \\
Sex & \\
Male & 58 \\
Female & 52 \\
Abnormal chest computed tomography findings & $97(88.2 \%)$ \\
Computed tomography findings & \\
$\quad$ Ground-glass opacities & $95(97.9 \%)$ \\
Consolidation & $57(58.8 \%)$ \\
Solid nodule & $1(1.0 \%)$ \\
Reticulonodular opacities & $1(1.0 \%)$ \\
Nodules with cavitation & 0 \\
Reticular/linear densities & $18(18.6 \%)$ \\
Septal thickening & $28(28.9 \%)$ \\
Bronchial wall thickening/dilatation & $13(13.4 \%)$ \\
Distribution of computed tomography findings & \\
Peripheral & $94(96.9 \%)$ \\
Single lobe & $17(17.5 \%)$ \\
Multilobar & $80(82.5 \%)$ \\
Unilateral & $20(20.6 \%)$ \\
Bilateral & $77(79.4 \%)$ \\
Other computed tomography findings & \\
Pleural effusion & $1(1.0 \%)$ \\
Mediastinal lymphadenopathy & $1(1.0 \%)$ \\
\hline
\end{tabular}

* Data are shown as No (\%), unless otherwise specified.

$77(79.4 \%)$ patients had bilateral involvement, and 80 $(82.5 \%)$ patients had multilobar involvement. Other imaging findings were considered atypical, such as pleural effusion in one (1.0\%) patient and mediastinal lymphadenopathy in one $(1.0 \%)$ patient. The observed imaging findings and patterns are consistent with the results from other published studies..$^{11,15,17-19}$

In March 2020, the Radiological Society of North America proposed, with endorsement by the Society of Thoracic Radiology and American College of Radiology, structured CT reporting for patients with COVID-19 pneumonia in order to disseminate common findings of the disease and decrease reporting variability.$^{20}$ Findings are classified into four categories: (1) typical appearance with (i) peripheral, bilateral GGOs with or without consolidation or intralobular lines ("crazy-paving" pattern), or (ii) multifocal GGOs of rounded morphology with or without consolidation or intralobular lines, or (iii) reverse halo sign or other findings of organising pneumonia; (2) indeterminate appearance with multifocal, diffuse, perihilar, or unilateral GGOs with or without consolidation lacking a specific distribution or few small GGOs that are non-rounded or non-peripheral; (3) atypical appearance; and (4) negative for pneumonia.

Based on this the Radiological Society of North America proposed reporting language for COVID-19 pneumonia, the majority of our patients had typical $(69 \%)$ or at least indeterminate appearances $(18 \%)$ on chest $\mathrm{CT}$, whereas few had atypical appearance $(0.9 \%)$ or were negative for pneumonia $(11.8 \%)$ [Table $2^{20}$. 


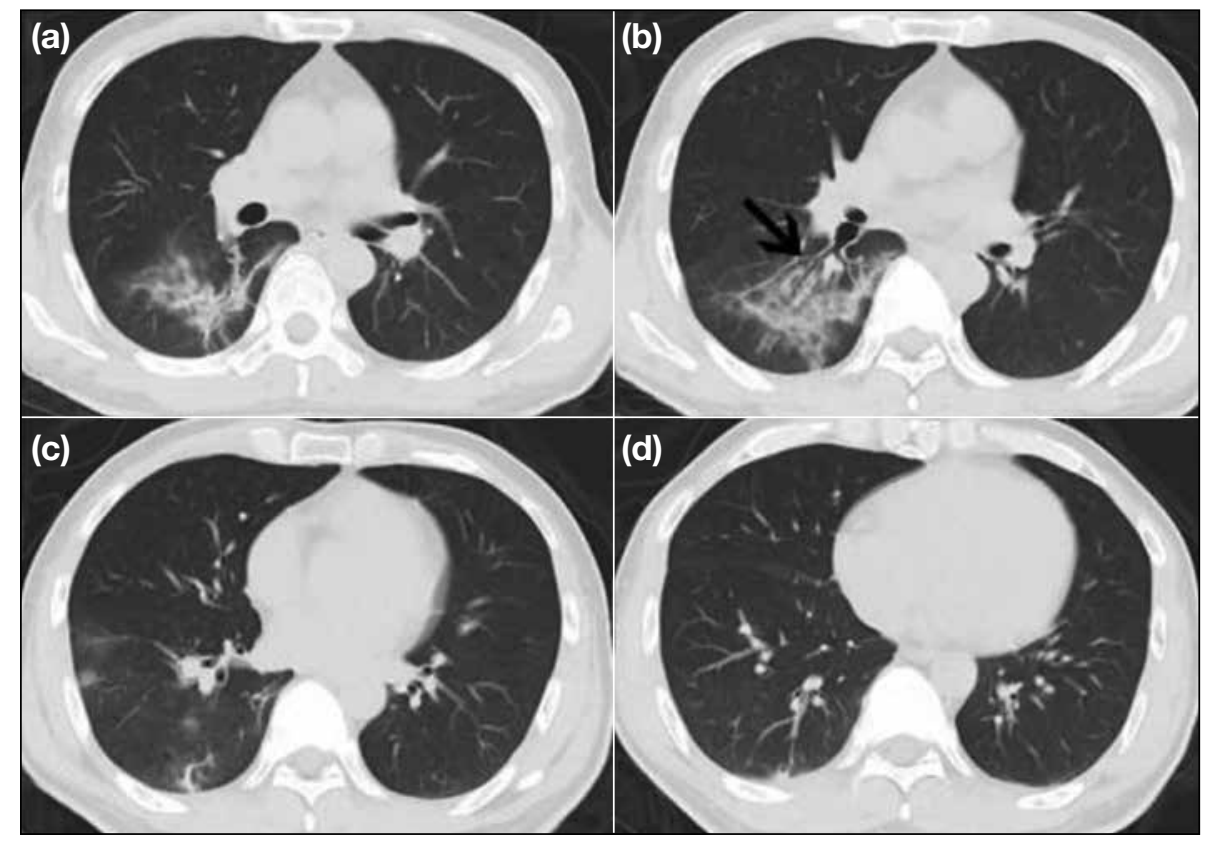

Figure 7. Indeterminate computed tomography imaging features of a 51-year-old man with coronavirus disease 2019. (a-d) Unenhanced, thin-section axial images of the lungs 12 days after the onset of symptoms showing unilateral, single lobar ground-glass opacities that lack a specific distribution and are non-rounded.
Table 2. Radiological Society of North America proposed reporting language for computed tomography findings related to coronavirus disease $2019 .{ }^{20}$

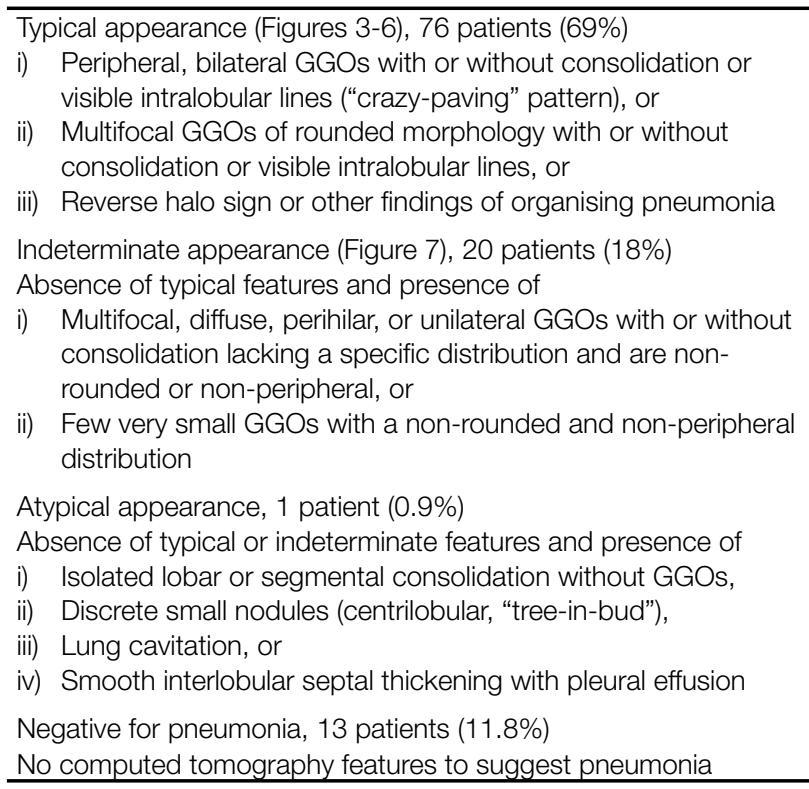

Abbreviation: $\mathrm{GGO}=$ ground-glass opacity.

\section{Follow-up Chest Computed Tomography}

When necessary, serial chest CT can reflect disease evolution and monitor treatment effects. ${ }^{22}$ In patients with COVID-19 who deteriorate, initial findings of small GGOs on chest CT become more extensive, and might grow larger with crazy-paving patterns and consolidation. ${ }^{22-24}$ In severe and critical patients, the occurrence rates of consolidation, linear opacities, crazypaving, and bronchial thickening increase, as well as the extent of lung involvement. ${ }^{23}$ Eventually the disease proceeds to 'white lung' appearance, seriously affecting the patient's lung function. ${ }^{24}$

For patients who eventually recover from pneumonia, the most severe features are found on CT imaging at approximately 10 days after the initial onset of symptoms. At approximately 14 days, radiological signs of improvement are seen. ${ }^{22} \mathrm{Ai}$ et al ${ }^{15}$ observed that chest CT improvement precedes negative RT-PCR test results in some patients.

Recent studies have revealed that COVID-19 shares similar CT features with organising pneumonia, most notably atoll signs and peripheral multilobar GGOs. Such similarities suggest the presence of secondary organising pneumonia in some patients with COVID-19. Secondary organising pneumonia was also documented in viral pneumonia caused by SARS-CoV, MERS-CoV, and influenza. Future histological correlations are required to confirm these associations. Because a small proportion of patients with organising pneumonia progress to pulmonary fibrosis (fibrosing organising pneumonia), follow-up CT imaging may be advisable for patients showing features of organising pneumonia. Organising pneumonia can be effectively managed by corticosteroids to prevent progression to fibrosis. ${ }^{25}$ 
According to a multinational consensus statement from the Fleischner Society, follow-up CT imaging is indicated for patients with functional impairment and hypoxaemia after recovery from COVID-19. The purpose of the follow-up CT imaging is to differentiate between causes for such pulmonary function impairment after recovery, whether it is due to sequelae of infection and mechanical ventilation or from a potentially treatable cause (e.g., organising pneumonia as mentioned above).$^{4}$

As stated above, not all patients with COVID-19 will undergo follow-up CT imaging. A single-centre review on the features of follow-up chest CT imaging revealed that among 70 patients who had chest CT scan after confirmation of SARS-CoV-2 infection, and 15 patients had a second chest CT scan. The mean ( \pm standard deviation) interval of the second chest CT after the first positive RT-PCR test was $21.9 \pm 5.8$ days. The mean interval between the first and second CT scan was $18.1 \pm 5.4$ days. The mean age of the patients was $56.1 \pm 16.7$ years. All patients had GGOs and consolidative changes with peripheral distribution on the initial scan. One (6.7\%) patient had complete resolution on the second CT, whereas other patients showed varying degrees of interval reductions in the GGOs and consolidative changes. All patients who underwent serial CT scans survived and were subsequently discharged home. Two patients underwent a third chest CT scan. One of them demonstrated features of secondary organising pneumonia (Figure 8). The other showed complete resolution on the third CT scan (Figure 9).

A recent report by Wang et $\mathrm{al}^{25}$ found that the majority of patients discharged home had residual disease on final CT scans. The number of days from symptom onset to discharge was median 24 days (range, 10-44 days). The CT changes peaked during illness days 6 to 11 after symptom onset. The increase in GGOs observed on serial scans by Wang et $\mathrm{al}^{25}$ was not observed in our reviewed

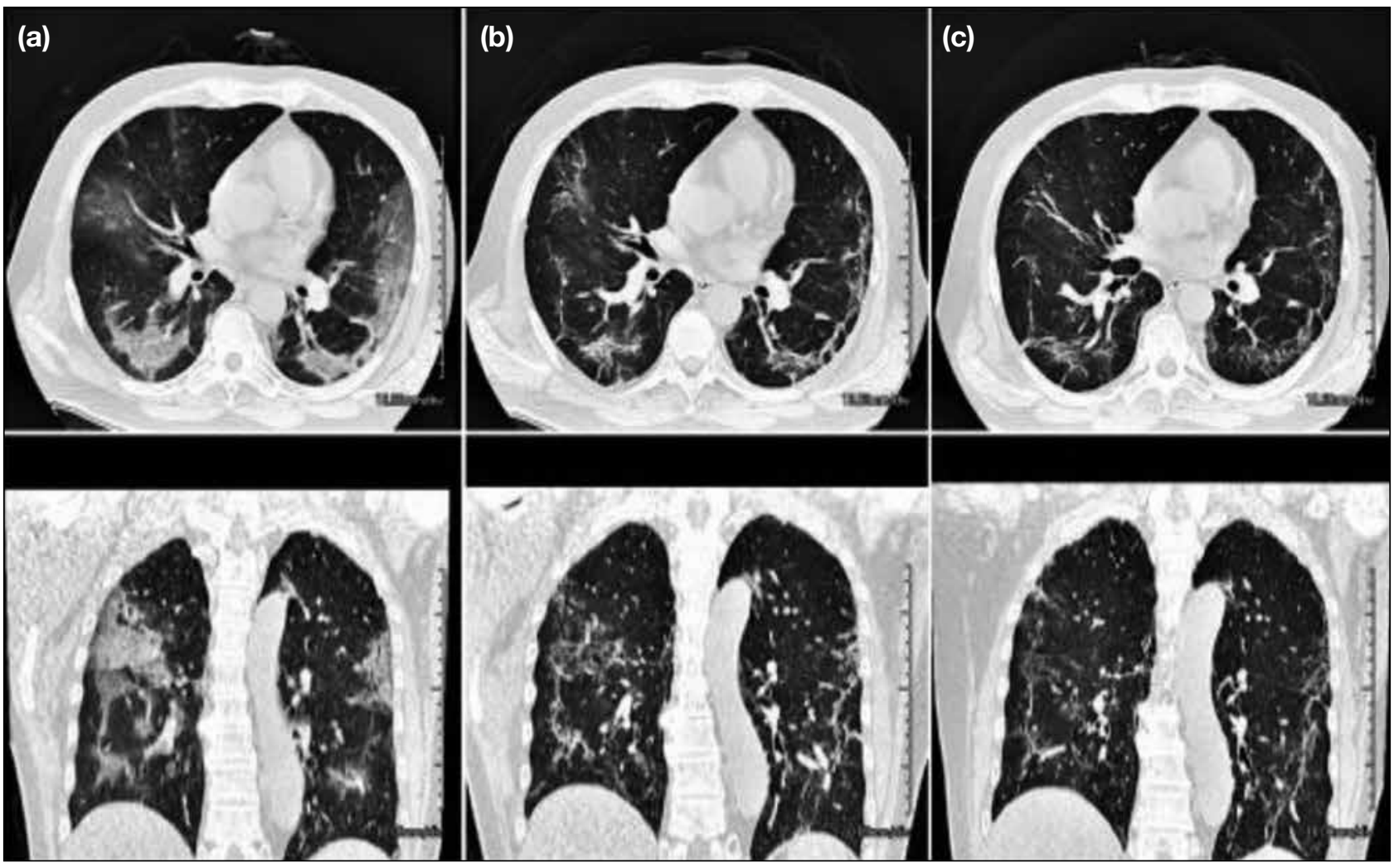

Figure 8. Serial chest computed tomography (CT) scans in a 56-year-old man with coronavirus disease 2019. Scan obtained on day 2 (a) after reverse-transcription polymerase chain reaction test confirmed severe acute respiratory syndrome coronavirus 2 infection chest CT scan showed peripheral ground-glass opacities and consolidations without zonal predominance. Scan obtained on day 14 (b) showed interval reduction of the mixed ground-glass opacities and consolidative changes. Scan obtained on day 22 (c) showing the consolidations became arched bands with shaded margins. It was likely the result of perilobular inflammation, which could represent features of perilobular fibrosis found in secondary organising pneumonia. The patient was discharged home on day 32 after two consecutive negative reversetranscription polymerase chain reaction tests. 


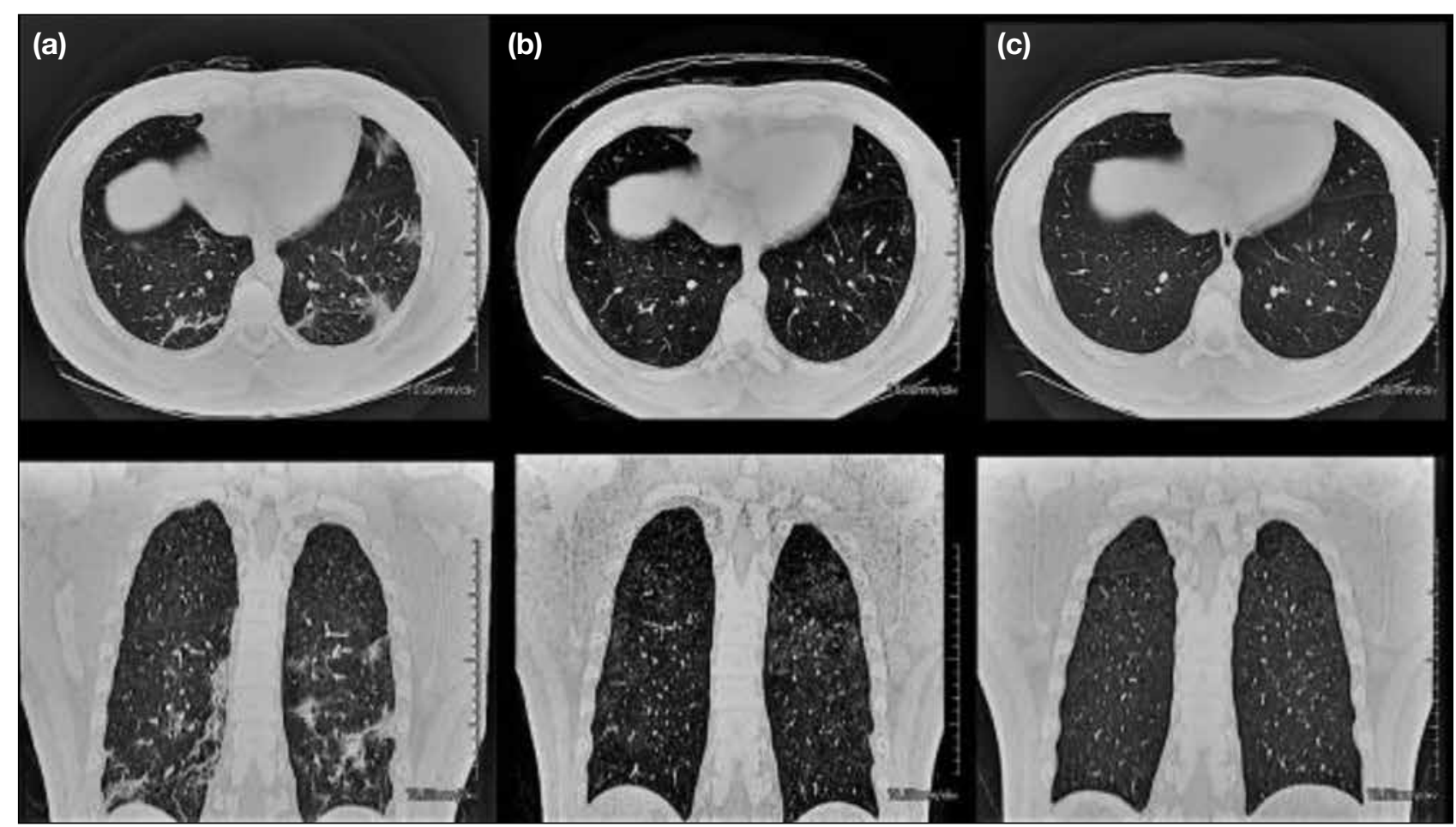

Figure 9. Serial chest computed tomography scans in a 39-year-old man with coronavirus disease 2019. Scan obtained on day 2 (a) after positive reverse-transcription polymerase chain reaction test for severe acute respiratory syndrome coronavirus 2 showed small peripheral consolidation and ground-glass opacities scattered in both middle and lower lobes. Scan obtained on day 14 (b) showing significant reduction of the consolidation and ground-glass opacities. Scan obtained on day 28 (c) showed complete resolution. The patient was discharged home on day 33 after two consecutive negative reverse-transcription polymerase chain reaction tests.

patients, which could be related to the longer median interval between scans of 17 days (range, 11-32 days) versus 6 days (range, 2-19 days).

\section{COMPARING IMAGING FEATURES AMONG COVID-19, SARS, AND MERS}

The COVID-19 pandemic in 2020, SARS outbreak in 2003, and MERS outbreak in 2012, are all caused by viruses belonging to the same Coronaviridae family. It is understandable that they can mimic each other on clinical grounds. Radiologically they share some common features but also have noticeable differences.

In the largest series of COVID-19 patients in China, abnormalities on radiographs were detected in up to $59.1 \%$ of patients at presentation. ${ }^{26}$ This is less than the reported radiographic detection rate of SARS and MERS (about 83\%). ${ }^{27,28}$ Among patients with radiological abnormalities, the distribution of changes most commonly involves the peripheral part of the lungs for all three infectious conditions. Unilateral abnormalities are more common in SARS and MERS than in COVID-19, where changes more frequently involve both lungs. Progression from focal unilateral peripheral lung involvement to bilateral multifocal lesions with upper lobes and perihilar involvement are associated with poor prognosis of severe cases in SARS (Figure 10) and MERS, whereas the prognostic value of radiographic changes remains unclear for COVID-19.

As with all viral pneumonias, the possible radiological patterns of parenchymal lung changes of these coronavirus infections are very similar. These include GGOs, consolidation, or a mixture of both. On CT imaging, interlobular septal thickenings and intralobular lines within the parenchymal changes are also welldescribed features, with possible progression into a crazy-paving pattern. ${ }^{27,29}$

It is also important to consider the important negative radiological findings of these infections. Cavitation, centrilobular nodules, mediastinal or hilar lymphadenopathy, and pleural effusion are not typical features in COVID-19 and SARS. ${ }^{29}$ In patients with MERS, pleural effusion was common $(33 \%)$ and is reported to be associated with poorer prognosis. ${ }^{27}$ 


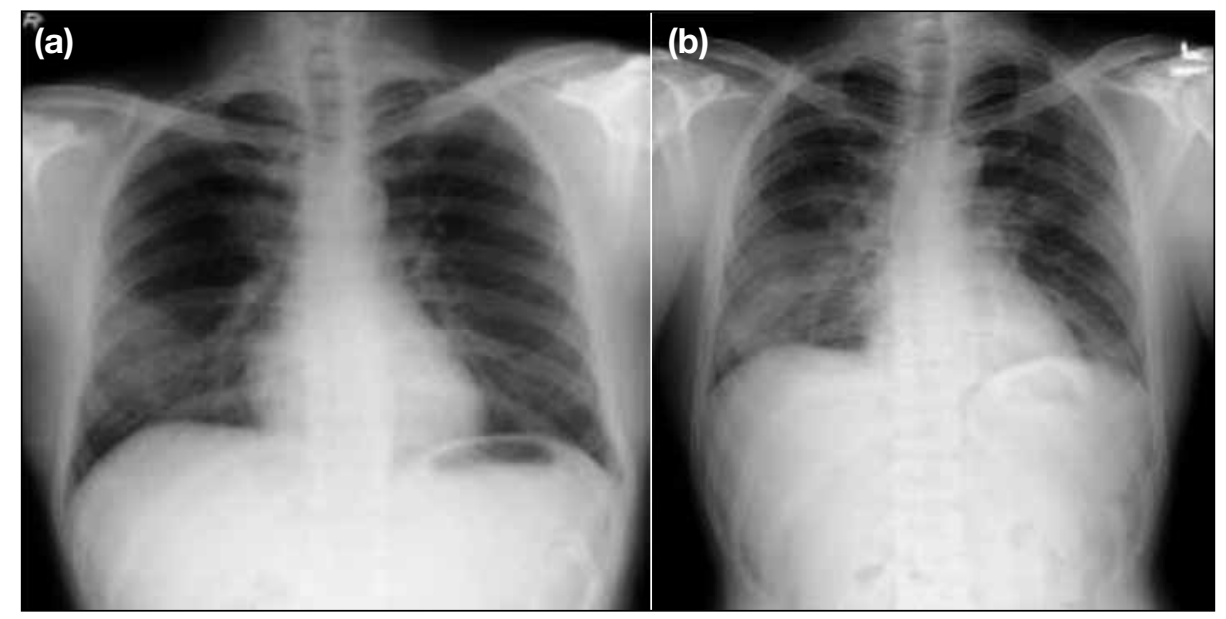

Figure 10. A 42-year-old patient with severe acute respiratory syndrome. (a) Plain chest X-ray taken on presentation, showing unilateral focal peripheral air space opacity at right lower zone. (b) Plain chest X-ray 5 days after presentation showing progression into bilateral multifocal air space opacities.
In long-term follow-up imaging for SARS patient with pneumonia, areas of air trapping due to damaged ciliated respiratory epithelium and lung fibrosis have been reported.$^{30}$ Likewise, fibrosis is a relatively common (33\%) feature among survivors of MERS. ${ }^{27}$ As it has only been a few months since COVID-19 was first reported, the long-term lung changes remain to be investigated with longer follow-up.

\section{POTENTIAL ROLE OF ARTIFICIAL INTELLIGENCE IN COVID-19 RESPONSE}

Recently, Li et al ${ }^{31}$ reported a deep-learning algorithm which can detect COVID-19 on chest CT images and differentiate it from community-acquired pneumonia. Covering up to 4000 multicentred chest CT examinations, this algorithm generated promising perexamination sensitivity and specificity of $90 \%$ and $96 \%$, respectively, with an average test time of $4.5 \mathrm{~s}$.

Artificial intelligence algorithms have assisted in early warning, diagnosing, triaging patients, and monitoring treatment response. For example, RADLogics allows quantification of opacities and provides a scoring system for patients with COVID-19 that correlates with disease severity. ${ }^{32}$ The Dutch University of Delft triages patients with COVID-19 based on CXR findings. ${ }^{33}$ For artificial intelligence to be an effective tool in responding to the COVID-19 pandemic, high-quality large populationbased datasets and worldwide concerted efforts are required for further development.

\section{ADAPTATION OF RADIOLOGY DEPARTMENTS DURING COVID-19 PANDEMIC}

The first confirmed case of SARS-CoV-2 infection in Hong Kong occurred on 23 January 2020 and the Hospital Authority raised the response level in public hospitals from "Serious" to "Emergency" on 25 January 2020. ${ }^{34}$ The first 20 patients with confirmed SARS-CoV-2 infection in Hong Kong were treated in an Infectious Disease Centre (IDC). Subsequent patients were treated in various acute public hospitals in the territory.

Modification and adaptation of the radiology department is essential in response to the COVID-19 pandemics. ${ }^{35,36}$ High-level infection control measures were implemented in radiology departments throughout Hong Kong. All patients with suspected and confirmed SARS-CoV-2 infection were treated in isolation wards in the IDC. Imaging facilities were provided in the IDC, including general radiography, CT, ultrasonography, and portable $\mathrm{C}$-arm radiography. The examination rooms were equipped with negative pressure to prevent the spread of infection. Most radiological examinations and procedures could be performed in the IDC without the need to transport the patient to main radiology department, thus reducing the risk of cross-infection. Portable radiographs were taken in the isolation wards with designated gown up and gown down areas for radiographers. The chest CT scans of patients with COVID-19 were grouped together in specific sessions with allowance of air exchange time (15 minutes for IDC CT with air change rate of $12 \mathrm{ACH}$; 
30 minutes for main CT with air change rate of $6 \mathrm{ACH}$ ) and room decontamination time between each patient.

Infection control measures were also implemented in the main radiology departments, including temperature screening of outpatients at hospital or department entrances and mask wearing for all patients, visitors, and staff. The radiological appointments for patients with COVID-19 awaiting RT-PCR results were deferred until the results were available. For patients with confirmed SARS-CoV-2 infection requiring radiological examinations for various clinical indications, $\mathrm{CT}$ was the preferred modality, rather than ultrasonography, owing to the shorter examination time and lower degree of physical contact between staff and patient.

Radiology service prioritisation, re-organisation, and manpower re-deployment were initiated to enhance the care of patients with COVID-19 in terms of imaging diagnosis, assessment of disease severity, and monitoring of progress and complication, as well as to maintain other essential diagnostic and interventional radiology services. This was achieved by rescheduling outpatient elective appointments and deploying more manpower to perform inpatient and urgent reporting and interventional procedures to speed up patient discharge. Examinations with higher risks such as barium enema and modified barium swallow were suspended or reduced. Face-to-face clinical radiological meetings and educational meetings were suspended and replaced by video conferencing, email, or telephone discussion. Some radiology departments also segregated radiologists and radiographers into "clean" and "dirty" teams ("dirty" being the colloquial term for "high risk for potential contamination or exposure to COVID-19") to limit cross-infection.

Staff training and engagement in infection control was another key measure. Personal infection prevention measures such as social distancing and vigilant handwashing were advocated. There was close liaison between the radiology department and the infection control team to ensure the adequate supply and distribution of personal protective equipment, enhancing infection control and staff morale. Timely communication with staff and other stakeholders including hospital management and infection control team was mediated through emails and social media apps to provide knowledge sharing and updates on information and policies. ${ }^{37,38}$

\section{CONCLUSION}

Radiological investigations play an important role in the diagnosis and management of COVID-19. The main imaging features on CXR include consolidation and GGOs, more often found in a bilateral, peripheral, and lower zone distribution. Compared with CXR, chest CT is more sensitive and provides superior delineation of the pulmonary involvement. The typical imaging findings of COVID-19 on chest CT are bilateral peripheral distribution of GGOs with or without consolidation. Interlobular septal thickening and intralobular lines creating a "crazy-paving" pattern may also be seen. In contrast, pleural effusion, lymphadenopathy and lung nodules are considered atypical. As it has only been a few months since the first case of COVID-19 was reported, further follow-up would be necessary to delineate the long-term radiological outcome in recovered patients with COVID-19.

Radiology departments of the public health sector have made adaptations and enhanced infection control. As more experience is accumulated, we are confident that radiology departments will continue to function well and assist the whole medical profession to respond to the COVID-19 pandemic.

\section{REFERENCES}

1. Wang L, Wang Y, Ye D, Liu Q. Review of the 2019 Novel Coronavirus (SARS-CoV-2) based on current evidence. Int J Antimicrob Agents. 2020 Mar 19. Epub ahead of print.

2. World Health Organization. Coronavirus disease 2019 (COVID-19) situation report-51. Available from: https://www.who.int/docs/ default-source/coronaviruse/situation-reports/20200311-sitrep-51covid-19.pdf?sfvrsn=1ba62e57_10. Accessed 11 Mar 2020.

3. World Health Organization. Coronavirus disease 2019 (COVID-19) situation report-90. Available from: https://www.who.int/docs/ default-source/coronaviruse/situation-reports/20200419-sitrep-90covid-19.pdf?sfvrsn=551d47fd_2. Accessed 19 Apr 2020.

4. Rubin GD, Ryerson CJ, Haramati LB, Sverzellati N, Kanne JP, Raoof $\mathrm{S}$, et al. The role of chest imaging in patient management during the COVID-19 pandemic: A multinational consensus statement from the Fleischner Society. Chest. 2020 Apr 7. Epub ahead of print.

5. Yoon SH, Lee KH, Kim JY, Lee YK, Ko H, Kim KH, et al. Chest radiographic and CT findings of the 2019 Novel Coronavirus Disease (COVID-19): analysis of nine patients treated in Korea. Korean J Radiol. 2020;21:494-500.

6. Ng MY, Lee EY, Yang J, Yang F, Li X, Wang H, et al. Imaging profile of the COVID-19 infection: radiologic findings and literature review. Radiol Cardiothorac Imaging. 2020 Feb 13. Epub ahead of print.

7. Wong HY, Lam HY, Fong AH, Leung ST, Chin TW, Lo CS, et al. Frequency and distribution of chest radiographic findings in COVID-19 positive patients. Radiology. 2020 Mar 27. Epub ahead of print. 
8. Arentz M, Yim E, Klaff L, Lokhandwala S, Riedo FX, Chong M, et al. Characteristics and outcomes of 21 critically ill patients with COVID-19 in Washington State. JAMA. 2020;323:1612-4.

9. Bandirali M, Sconfienza LM, Serra R, Brembilla R, Albano D, Pregliasco FE, et al. Chest radiograph findings in asymptomatic and minimally symptomatic quarantined patients in Codogno, Italy during COVID-19 pandemic. Radiology. 2020;295:E7.

10. Kim ES, Chin BS, Kang CK, Kim NJ, Kang YM, Choi JP, et al. Clinical course and outcomes of patients with severe acute respiratory syndrome coronavirus 2 infection: a preliminary report of the first 28 patients from the Korean cohort study on COVID-19. J Korean Med Sci. 2020;35:e142.

11. Salehi S, Abedi A, Balakrishnan S, Gholamrezanezhad A. Coronavirus disease 2019 (COVID-19): a systematic review of imaging findings in 919 patients. AJR Am J Roentgenol. 2020 Mar 14. Epub ahead of print.

12. Zhou F, Yu T, Du R, Fan G, Liu Y, Liu Z, et al. Clinical course and risk factors for mortality of adult inpatients with COVID-19 in Wuhan, China: a retrospective cohort study. Lancet. 2020;395:1054-62.

13. Kim D, Quinn J, Pinsky B. Shah NH, Brown I. Rates of co-infection between SARS-CoV-2 and other respiratory pathogens. JAMA. 2020 Apr 15. Epub ahead of print.

14. Fang Y, Zhang H, Xie J, Lin M, Ying L, Pang P, et al. Sensitivity of chest CT for COVID-19: comparison to RT-PCR. Radiology. $2020 \mathrm{Feb} 19$. Epub ahead of print.

15. Ai T, Yang Z, How H, Zhan C, Chen C, Lv W, et al. Correlation of chest CT and RT-PCR testing in coronavirus disease 2019 (COVID-19) in China: a report of 1014 cases. Radiology. 2020 Feb 26. Epub ahead of print.

16. Inui S,Fujikawa A,JitsuM,KunishimaN, Watanabe S,SuzukiY, et al. Chest CT findings in cases from the cruise ship "Diamond Princess" with coronavirus disease 2019 (COVID-19). Radiol Cardiothorac Imaging. 2020 Mar 17. Epub ahead of print.

17. Wen Z, Chi Y, Zhang L, Liu H, Du K, Li Z, et al. Coronavirus disease 2019: initial detection on chest CT in a retrospective multicenter study of 103 Chinese subjects. Radiol Cardiothorac Imaging. 2020 Apr 6. Epub ahead of print.

18. XuX, YuC, QuJ,Zhang L,Jiang S,Huang D, etal.Imaging and clinical features of patients with 2019 novel coronavirus SARS-CoV-2. Eur J Nucl Med Mol Imaging.2020;47:1275-80.

19. Bernheim A, Mei X, Huang M, Yang Y, Fayad ZA, Zhang N, et al. Chest CT findings in coronavirus disease-19 (COVID-19): relationship to duration of infection. Radiology. 2020;295:200463.

20. Simpson S, Kay FU, Abbara S, Bhalla S, Chung JH, Chung M, et al. Radiological Society of North America Expert Consensus Statement on reporting chest CT findings related to COVID-19. endorsed by the Society of Thoracic Radiology, the American College of Radiology, and RSNA. Radiol Cardiothorac Imaging. 2020 Mar 25. Epub ahead of print.

21. Ye Z, Zhang Y, Wang Y, Huang Z, Song B, et al. Chest CT manifestations of new coronavirus disease 2019 (COVID-19): a pictorial review. Eur Radiol. 2020 Mar 19. Epub ahead of print.

22. Pan F, Ye T, Sun P, Gui S, Liang B, Li L, et al. Time course of lung changes on chest $\mathrm{CT}$ during recovery from coronavirus disease 2019 (COVID-19). Radiology. 2020;295:715-21.

23. Li K, Wu J, Wu F, Guo D, Chen L, Fang Z, et al. The clinical and chest CT features associated with severe and critical COVID-19 pneumonia. Invest Radiol. 2020;55:327-31.

24. Pan Y, Guan H, Zhou S, Wang Y, Li Q, Zhu T, et al. Initial $\mathrm{CT}$ findings and temporal changes in patients with the novel coronavirus pneumonia (2019-nCoV): a study of 63 patients in Wuhan, China. Eur Radiol. 2020 Feb 13. Epub ahead of print.

25. Wang Y, Dong C, Hu Y, Li C, Ren Q, Zhang X, et al. Temporal changes of CT findings in 90 patients with COVID-19 pneumonia: a longitudinal study. Radiology. 2020 Mar 19. Epub ahead of print.

26. Guan WJ, Ni ZY, Hu Y, Liang WH, Ou CQ, He JX, et al. Clinical characteristics of coronavirus disease 2019 in China. N Engl J Med. 2020;382:1708-20.

27. Das KM, LeeEY, Langer RD, Larsson SG. Middle East Respiratory Syndrome coronavirus: what does a radiologist need to know? AJR Am J Roentgenol. 2016;206:1193-201.

28. Hosseiny M, Kooraki S, Gholamrezanezhad A, Reddy S, Myers L. Radiology perspective of coronavirus disease 2019 (COVID-19): lessons from severe acute respiratory syndrome and Middle East respiratory syndrome. AJR Am J Roentgenol. 2020;214:107882.

29. Antonio GE, Wong KT, Hui DS, Lee N, Yuen EH, Wu A, et al Imaging of severe acute respiratory syndrome in Hong Kong. AJR Am J Roentgenol. 2003;181:11-7.

30. Chang YC, Yu CJ, Chang SC, Galvin JR, Liu HM, Hsiao CH, et al. Pulmonary sequelae in convalescent patients after severe acute respiratory syndrome: evaluation with thin-section CT. Radiology. 2005;236:1067-75

31. Li L, Qin L, Xu Z, Yin Y, Wang X, Kong B, et al. Artificial intelligence distinguishes COVID-19 from community acquired pneumonia on chest CT. Radiology. 2020 Mar 19. Epub ahead of print.

32. Gozes O, Frid-Adar M, Greenspan H, Browning PD, Zhang H, $\mathrm{Ji}$ W, et al. Rapid AI development cycle for the coronavirus (COVID-19) pandemic: Initial results for automated detection \& patient monitoring using deep learning CT image analysis. arXiv . [Preprint] 2003. Available from: https://arxiv.org/abs/2003.05037. Accessed 30 Mar 2020.

33. Delft Imaging. Triage for COVID-19 using artificial intelligence on chest X-rays. Available from: https://www.delft.care/cad4covid/. Accessed 30 Mar 2020.

34. Hospital Authority, Hong Kong SAR Government. Hospital Authority activates Emergency Response Level [press release]. 2020 Jan 25. Available from: https://www8.ha.org.hk/qmh/ index_doc/eng.pdf. Accessed 25 Jan 2020

35. Mossa-Basha M, Meltzer CC, Kim DC, Tuite MJ, Kolli KP, Tan BS. Radiology department preparedness for COVID19: Radiology Scientific Expert Panel. Radiology. 2020 Mar 16. Epub ahead of print.

36. Goh Y, Chua W, Lee JK, Leng Ang BW, Liang CR, Tan CA, et al. Operational strategies to prevent COVID-19 spread in radiology: experience from a Singapore radiology department after severe acute respiratory syndrome. J Am Coll Radiol. 2020 Apr 3. Epub ahead of print.

37. Huang Z, Zhao S, Li Z. Chen W, Zhao L, Deng L, et al. The battle against coronavirus disease 2019 (COVID-19): emergency management and infection control in a radiology department. J Am Coll Radiol. 2020 Mar 24. Epub ahead of print.

38. Lai TS, Yu WC. The lessons of SARS in Hong Kong. Clin Med (Lond). 2010;10:50-3. 